\title{
PROTON POLARIZATION IN NEUTRAL PION PHOTO-PRODUCTION
}

\author{
K. WIJESOORIYA \\ Argonne National Laboratory, Argonne, IL, 60439, USA \\ E-mail: krishniw@jlab.org \\ FOR THE JEFFERSON LAB HALL A COLLABORATION
}

\begin{abstract}
We present measurements of recoil proton polarization for ${ }^{1} H(\vec{\gamma}, \vec{p}) \pi^{0}$ in and above the resonance region. These are the first data in this reaction for polarization transfer with circularly polarized photons. The results are compared to phase shift analyses and quark model calculations.
\end{abstract}

\section{INTRODUCTION}

At photon energies below about $2 \mathrm{GeV}$, corresponding to $W=\sqrt{s}$ below $2.15 \mathrm{GeV}, \pi^{0}$ photo-production is dominated by the production and decay of baryon resonances, indicated by structure in the cross section ${ }^{1}$ and polarization observables. There are extensive data for only a few spin observables, and only for $E_{\gamma} \leq 1.5 \mathrm{GeV}$. We present recoil proton polarization measurements which extend across the resonance region, up to $W \gg$ $2 \mathrm{GeV}$, at large scattering angles and four momentum transfers.

Above the resonance region, the cross section follows the constituent counting rules ${ }^{2}$, which can be derived from perturbative QCD (pQCD), as is the case for a number of exclusive photo-reactions ${ }^{3,4}$. Hadron helicity conservation ${ }^{5}$ (HHC) is also taken to be a consequence of pQCD, although this has come under question due to the lack of $\mathrm{HHC}$ in hadronic reactions ${ }^{6,7,8}$. In hadronic photo-reactions, HHC may hold if a single photon interacts only with a single quark in the target ${ }^{9}$, and if orbital angular momenta may be neglected. A recent paper by Miller and Frank ${ }^{10}$ suggests that helicity conservation is not satisfied for exclusive processes involving protons. HHC predicts that the induced polarization $p_{y}$ and the transferred polarization $C_{x^{\prime}}$ c.m. vanish. In the one photo-reaction tested, deuteron photodisintegration ${ }^{11}$, helicity conservation was not valid.

The experiment ran in Hall A of the Thomas Jefferson National Accelerator Facility (JLab). Space limitations preclude discussion of the details, 
which may be found in Ref. ${ }^{12}$.

\section{MODELS}

Predictions for $\mathrm{H}(\vec{\gamma}, \vec{p}) \pi^{0}$ come from the phase shift analysis codes SAID ${ }^{13}$ and MAID ${ }^{14}$, and from quark model ${ }^{15}$, and pQCD ${ }^{16}$ calculations. In SAID, both an energy-dependent and a set of single energy partial wave analyses of single-pion photo-production data are performed. These analyses extend from threshold to $2.0 \mathrm{GeV}$ in laboratory photon energy. Photo decay amplitudes are extracted from Breit-Wigner fits for the baryon resonances within this energy range. The MAID model contains Born terms, vector mesons and nucleon resonances up to the third resonance region ( $P_{33}(1232), P_{11}(1440), D_{13}(1520), S_{11}(1535), F_{15}(1680)$ and $\left.D_{33}(1700)\right)$. This model is fitted to data up to $E_{\gamma}=1.25 \mathrm{GeV}$. The resonance contributions are included taking into account unitarity to provide the correct phases of the pion photo-production multipoles.

Afanasev et al. ${ }^{15}$ use a pQCD approach for large transverse momenta, $p_{T}$, where mesons are directly produced by short range processes - see also Ref. ${ }^{17}$. This approach is similar to the factorization approach ${ }^{17}$ used to describe Compton scattering from the proton. The calculation assumes helicity conservation, which leads to the vanishing of $p_{y}$ and $C_{x^{\prime}}$ c.m. In the lab, $C_{x^{\prime}}$ does not generally vanish as it has contributions from both $C_{x^{\prime}}$ c.m. and $C_{z^{\prime} \text { c.m. }}$. This gives a simple result for exclusive photo-production of neutral pions:

$$
\begin{aligned}
& p_{y}=C_{x^{\prime} c . m .}=0 \\
& C_{z^{\prime} \text { c.m. }}=\frac{s^{2}-u^{2}}{s^{2}+u^{2}} .
\end{aligned}
$$

This model assumes that the polarization of the struck quark is the same as the polarization of the outgoing proton, but wave function effects can reduce the polarization. Farrar et al. ${ }^{16}$ use pQCD to calculate all lowestorder $\left(\alpha_{s}^{3}\right)$ Feynman diagrams. They assume HHC, so $p_{y}=C_{x^{\prime} c . m}$. $=$ $0 ; C_{z^{\prime} c . m}$. is constant at fixed $\theta_{c . m .}$. The exact magnitude of $C_{z^{\prime} c . m}$. can be large, but it depends on the hadronic distribution amplitudes. The calculations shown here used the asymptotic distribution amplitudes for both the proton and the pion. These pQCD approaches underpredict the cross section, and are not expected to work until $-t$ is very large.

\section{RESULTS}

Figure 1 compares our data for $p_{y}$ to previous measurements - see Ref. ${ }^{12}$ for a complete list of references - and theoretical predictions ${ }^{13,14}$. Our 


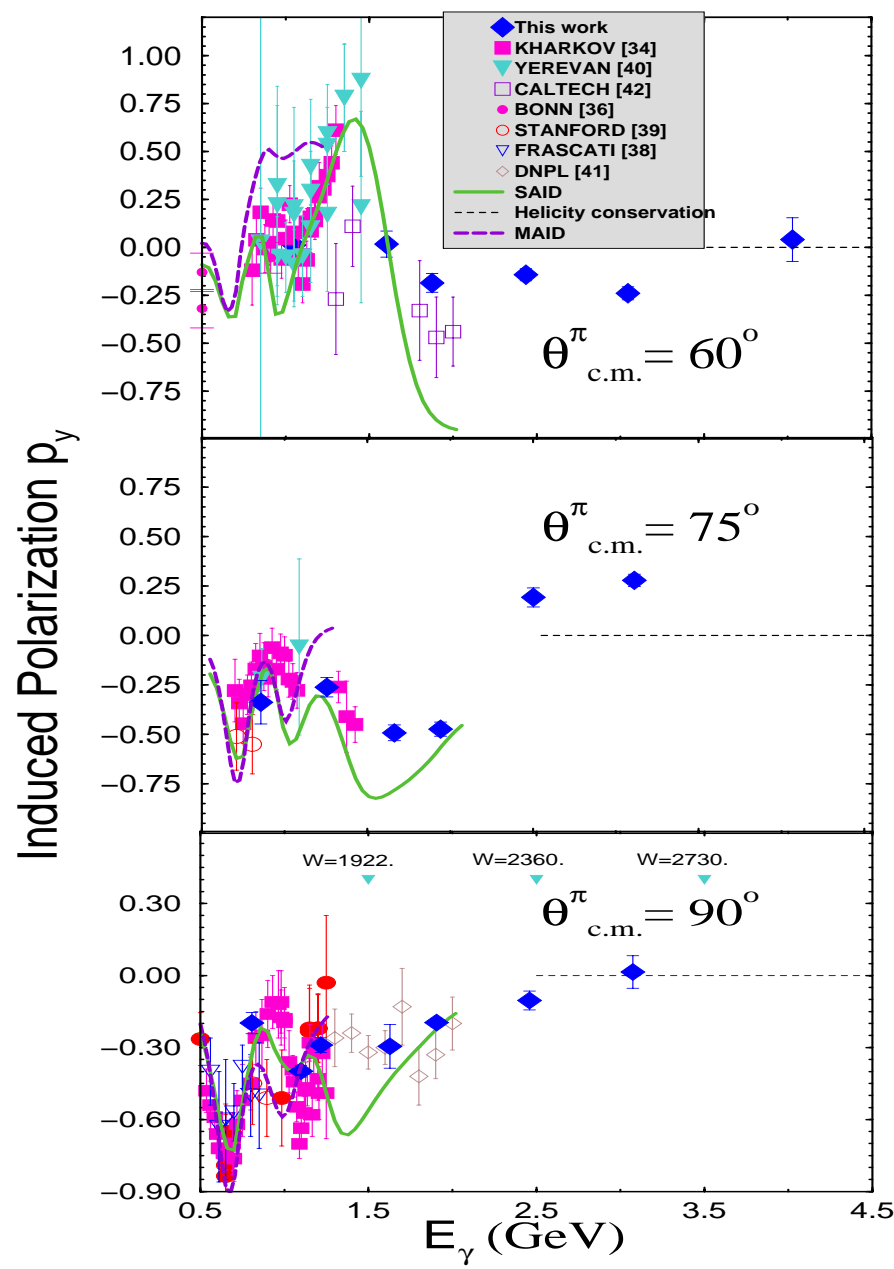

Figure 1. Induced polarization $p_{y}$ at three c.m. angles. Only statistical uncertainties are shown. The curves are described in the text.

low-energy data agree well with the world data. At higher energy, the data follow the trend predicted by SAID, but not by MAID. There is no general indication of an approach to helicity conservation, $p_{y} \rightarrow 0$; similarly, we find that $C_{x^{\prime} \text { c.m. }}$ does not tend to vanishing.

The longitudinal in-plane polarization transfer $C_{z^{\prime}}$, shown in Fig. 2, does not show large polarizations as predicted in Ref. ${ }^{15}$, nor does it appear to reach a constant value at each angle, as predicted by Ref. ${ }^{16}$. The data agree reasonably well with the phase shift analysis curves, SAID and MAID, at the lowest energies, but the agreement tends to increasingly deteriorate 


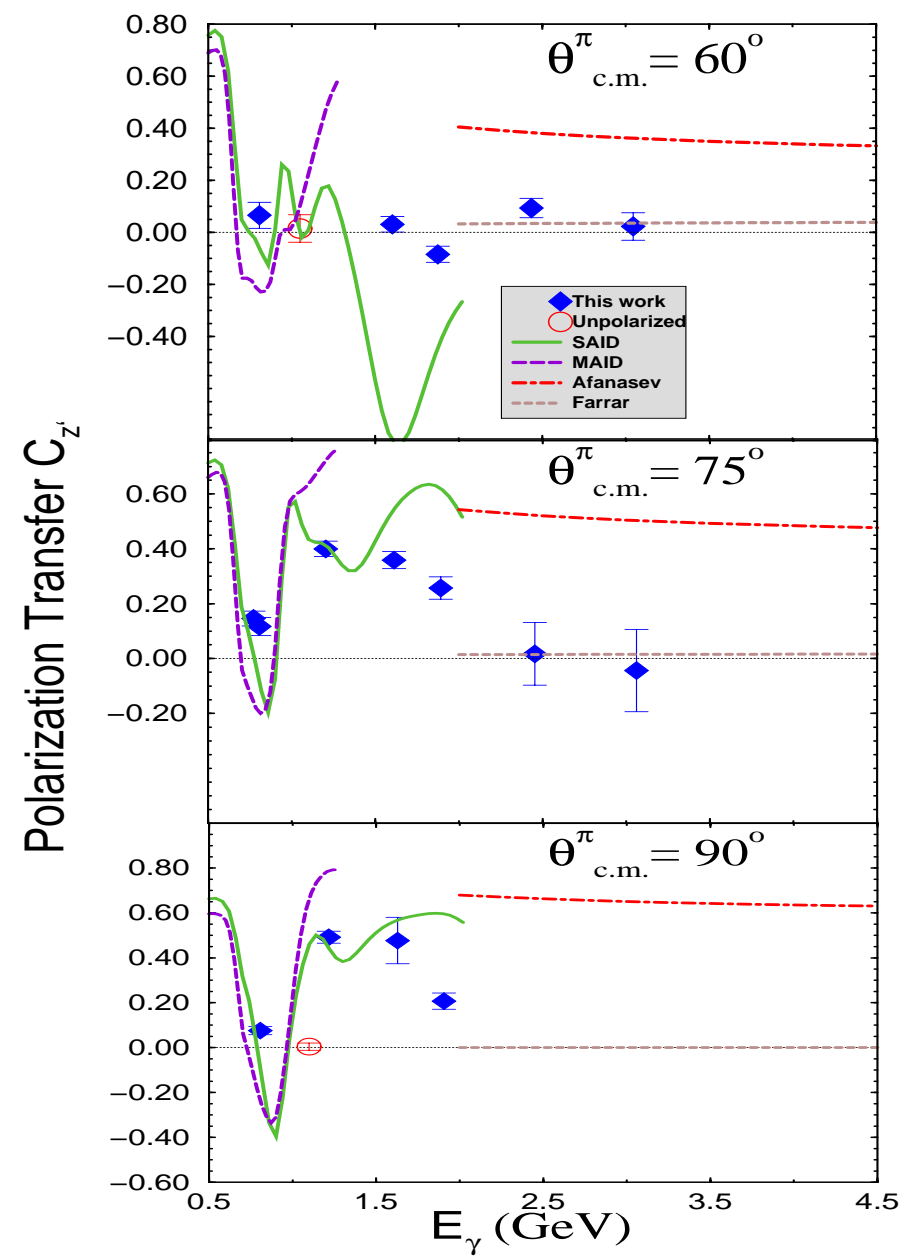

Figure 2. Polarization transfer $C_{z^{\prime}}$ at three c.m. angles. Only statistical uncertainties are shown. The curves are described in the text.

with energy. There is insufficient space to show that similar observations can be made for $C_{x^{\prime}}$. Data with unpolarized beam at $\mathrm{E}_{\gamma}=1.1 \mathrm{GeV}$, are consistent with zero, as expected.

Figure 3 shows some angular distributions for $p_{y}$. The data at $2.5 \mathrm{GeV}$ suggests a strong oscillatory behavior, and the $\sin (12 \theta)$ curve indicates high partial waves in either the background or resonances. More finely binned angular distributions are evidently needed. The strong angular dependence appears to persist up to at least $3.1 \mathrm{GeV}$, suggesting that resonances are needed to explain the data, and quark models which sum over the reso- 


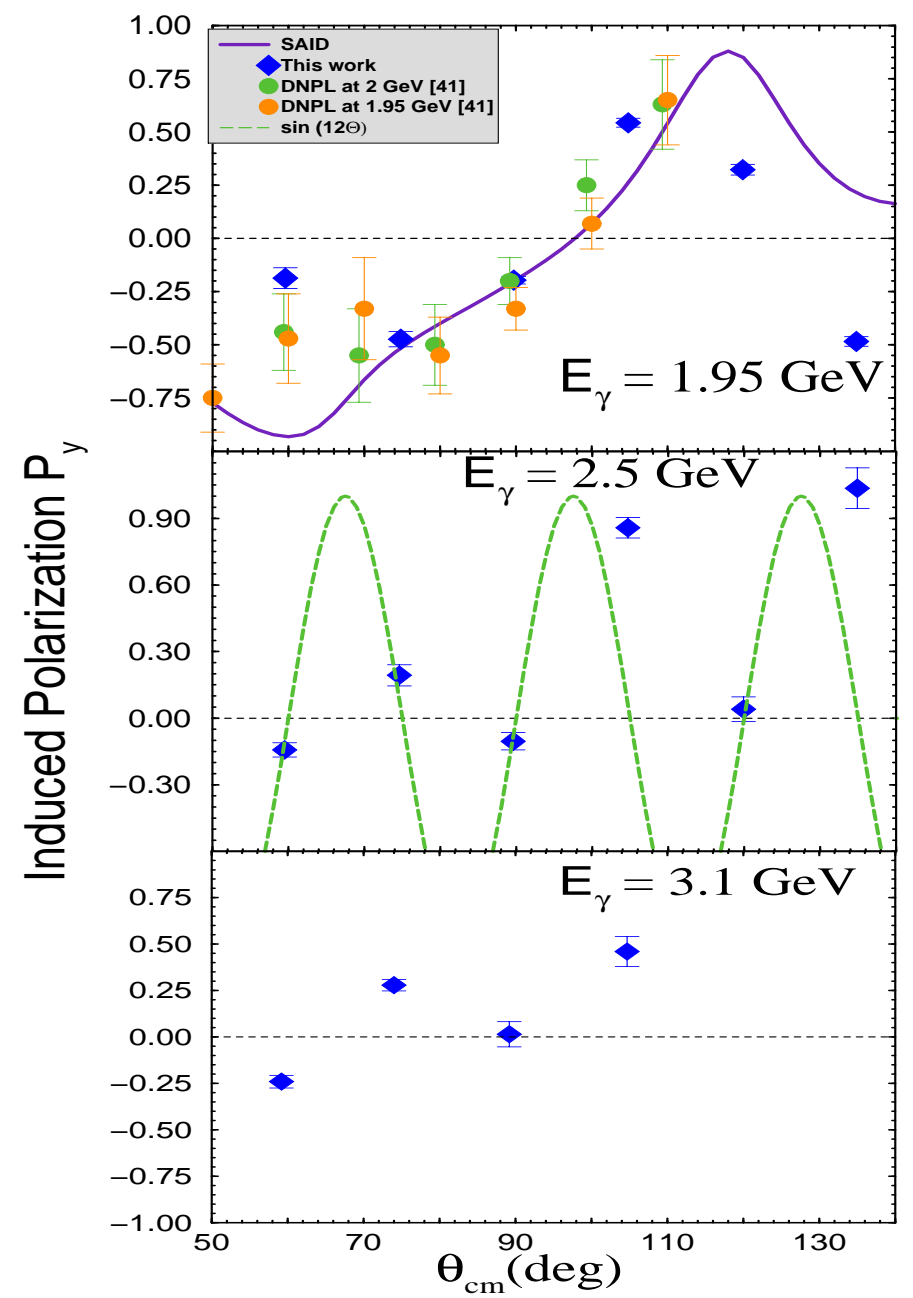

Figure 3. Angular distributions of induced polarization $p_{y}$ at three beam energies. The curves are described in the text.

nances will be unable to reproduce the polarizations.

\section{CONCLUSIONS}

Our induced polarizations extend the world data for $\pi^{0}$ photo-production from the proton to much higher photon energies. Our data agree reasonably well with previous data and with the SAID and MAID phase shift analyses. We present the first data set for polarization transfer observables $C_{x^{\prime}}$ and $C_{z^{\prime}}$ for the ${ }^{1} H(\vec{\gamma}, \vec{p}) \pi^{0}$ reaction. The nonzero nature of $p_{y}$ and 
the polarization transfer component, $C_{x^{\prime} c . m}$., show that hadron helicity is not conserved. The strong angle dependence of $p_{y}$ indicates interference between resonances and the non-resonant background. This confirms the importance of polarization observables as a powerful tool to look for resonance effects. A data set finely binned in angle would be extremely useful in advancing the theoretical analysis.

The data do not agree with existing quark model calculations, and thus do not support possible factorization approaches in these kinematics. These data, with a large angle and energy coverage, should help to develop a better understanding of photo-pion production mechanisms in the $\mathrm{GeV}$-region.

\section{Acknowledgements}

I acknowledge many constructive conversations with R. J. Holt and R. Gilman. This work was supported in part by the United States National Science Foundation and Department of Energy; the Southeastern Universities Research Association operates the Thomas Jefferson National Accelerator Facility under Department of Energy contract DE-AC05-84ER40150.

\section{References}

1. Data and references can be found in Compilation of Pion Photoproduction Data, D. Menze, W. Pfeil, and R. Wilcke, Physikalisches Institut der Universitat Bonn (1977).

2. S.J. Brodsky and G.R. Farrar, Phys. Rev. Lett. 311153 (1973); V. Matveev et al., Nuovo Cimento Lett. 7, 719 (1973).

3. R.L. Anderson et al., Phys. Rev. D 14679 (1976).

4. See E.C. Schulte et al., Phys. Rev. Lett. 87102302 (2001), and references therein.

5. See S.J. Brodsky and G.P. Lepage, Phys. Rev. D 242848 (1981), and references therein.

6. P.V. Landshoff, Phys. Rev. D 101024 (1974).

7. T. Gousset, B. Pire, and J.P. Ralston, Phys. Rev. D 531202 (1996).

8. C. Carlson and M. Chachkhunashvili, Phys. Rev. D 452555 (1992).

9. A. Afanasev, C. Carlson, and C. Wahlquist, Phys. Rev. D 61034014 (2000).

10. G.A. Miller, M.R. Frank, arXiv:nucl-th/0201021.

11. K. Wijesooriya et al., Phys. Rev. Lett. 862975 (2001).

12. K. Wijesooriya et al., accepted by Phys. Rev. C.

13. R.A. Arndt et al., Phys. Rev. C 53430 (1996); R.A. Arndt et al., Phys. Rev. C 56577 (1997).

14. D. Drechsel et al., Nucl. Phys. A 645145 (1999); Version MAID 2000.

15. A. Afanasev, C. Carlson, and C. Wahlquist, Phys. Lett. B 398393 (1997).

16. G.R. Farrar, K. Huleihel, and H. Zhang, Nucl. Phys. B 349655 (1991).

17. A.V. Radyushkin, Phys. Rev. D 5811408 (1998); M. Diehl, Eur. Phys. J. C 8409 (1999); H.W. Huang and P. Kroll, Eur. Phys. J. C 17423 (2000). 